\title{
Argumentation for Knowledge Representation, Conflict Resolution, Defeasible Inference and Its Integration with Machine Learning
}

Luca Longo

Technological University Dublin, luca.longo@tudublin.ie

Follow this and additional works at: https://arrow.tudublin.ie/scschcomcon

Part of the Artificial Intelligence and Robotics Commons

\section{Recommended Citation}

Longo L. (2016) Argumentation for Knowledge Representation, Conflict Resolution, Defeasible Inference and Its Integration with Machine Learning. In: Holzinger A. (eds) Machine Learning for Health Informatics. Lecture Notes in Computer Science, vol 9605. Springer, Cham. DOI: 10.1007/978-3-319-50478-0_9

This Book Chapter is brought to you for free and open access by the School of Computer Sciences at ARROW@TU Dublin. It has been accepted for inclusion in Conference papers by an authorized administrator of ARROW@TU Dublin. For more information, please contact arrow.admin@tudublin.ie, aisling.coyne@tudublin.ie,gerard.connolly@tudublin.ie.

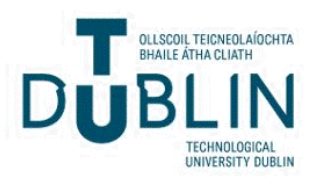




\title{
Argumentation for Knowledge Representation, Conflict Resolution, Defeasible Inference and Its Integration with Machine Learning
}

\author{
Luca Longo ${ }^{(凶)}$ \\ School of Computing, College of Sciences and Health, \\ Dublin Institute of Technology \\ ADAPT: \\ The Global Centre of Excellence for Digital Content and Media Innovation, \\ Dublin, Republic of Ireland \\ luca.longo@dit.ie
}

\begin{abstract}
Modern machine Learning is devoted to the construction of algorithms and computational procedures that can automatically improve with experience and learn from data. Defeasible argumentation has emerged as sub-topic of artificial intelligence aimed at formalising common-sense qualitative reasoning. The former is an inductive approach for inference while the latter is deductive, each one having advantages and limitations. A great challenge for theoretical and applied research in $\mathrm{AI}$ is their integration. The first aim of this chapter is to provide readers informally with the basic notions of defeasible and non-monotonic reasoning. It then describes argumentation theory, a paradigm for implementing defeasible reasoning in practice as well as the common multi-layer schema upon which argument-based systems are usually built. The second aim is to describe a selection of argument-based applications in the medical and health-care sectors, informed by the multi-layer schema. A summary of the features that emerge from the applications under review is aimed at showing why defeasible argumentation is attractive for knowledgerepresentation, conflict resolution and inference under uncertainty. Open problems and challenges in the field of argumentation are subsequently described followed by a future outlook in which three points of integration with machine learning are proposed.
\end{abstract}

Keywords: Defeasible reasoning - Argumentation • Conflict resolution • Knowledge-representation $\cdot$ Interactive machine learning $\cdot$ Medicine

\section{Introduction}

The fast-growing field of Machine Learning (ML) is devoted to the construction of algorithms and computational procedures that can automatically improve with experience and learn from data. Although ML is increasing in popularity with a plethora of applications in several fields, and it has proved to be useful in the identification and extraction of meaningful patterns of data and rules, 
it is often based upon algorithms that implement quantitative manipulation of training data. These algorithms are frequently used as 'black-boxes' and the inference process that lead to the quantitative output is neglected. In the last two decades, Defeasible Reasoning (DR) has emerged as sub-topic of artificial intelligence (AI) aimed at formalising common-sense qualitative reasoning. This type of reasoning is often performed in contexts characterised by high uncertainty, such as medicine and health care, where available information is usually fragmented, partial, conflicting, noisy and multi-dimensional. Defeasible reasoning can be combined to machine learning inference techniques and a great challenge for theoretical and applied research in AI is their integration. This challenge is highly connected to the notion of interactive Machine Learning (iML) $[1,2]$ being proposed in this book. In particular, as Fig. 1 depicts, on one hand machine learning might support defeasible reasoning by providing it with quantitative evidence for enhancing reasoning processes. On the other hand, defeasible reasoning might contribute to extend and enhance the inferential mechanisms behind machine learning techniques with more qualitative and transparent reasoning and by incorporating intelligence and argumentative capacity. The integration of these two subfields of AI is likely to impact and contribute to design and develop intelligence agents with greater knowledge extraction, predictive power as well as argumentative and reasoning capabilities [3]. Machine learning is a more mature branch of research within artificial intelligence than formal defeasible reasoning. Therefore the main focus of this chapter is on the latter paradigm with emphasis on argumentation theory and argument-based systems, the computational approaches to implement defeasible reasoning in practice. The rest of this document is organised as it follows. Firstly, a glossary describes the core definitions and terms of this desk research. Argumentation theory is subsequently introduced with an emphasis on its role in defeasible reasoning. This is complemented by a detailed description of the multi-layered pattern upon which argument-based systems are usually built. An overview of practical applications of argumentation in clinical domains is then presented followed by a description of the main features and advantages of defeasible reasoning and argumentation theory in decision-making and knowledge representation. Open problems and challenges in applied research are then discussed and a summary concludes this chapter with a future outlook for argumentation and its integration with machine learning.

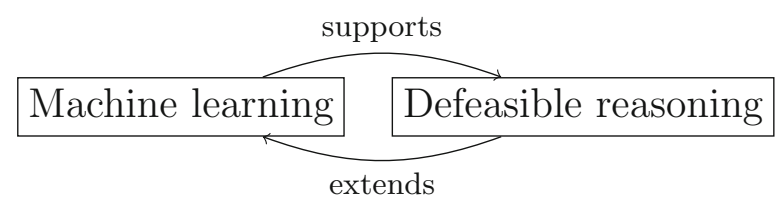

Fig. 1. Interaction of argumentation and machine learning 


\section{Glossary and Key Terms}

Machine learning $(M L)$ : subfield of computer science devoted to the design of computational procedures able to learn from and perform prediction of data.

Default knowledge: kn owledge routinely employed by humans in a reasoning process even if the preconditions for its application are only partially known.

Defaults: specific inference rules employed in default knowledge.

Monotonicity: property of a reasoning process in which conclusions are not affected by new pieces of evidence and, as a consequence, the set of available conclusions monotonically increases.

Non-monotonicity: property of a reasoning process in which conclusions can be retracted in the light of new pieces of evidence, and as a consequence, the set of available conclusions can decrease in cardinality.

Defeasible reasoning $(D R)$ : a type of reasoning with the non-monotonicity property based upon reasons that are defeasible. This reasoning does not produce a complete and final demonstration of a claim, instead it acknowledges corrigibility and fallibility of a conclusion.

Argumentation theory (AT): a multidisciplinary area of artificial intelligence that provides state-of-the-art computational models of defeasible reasoning.

Argument: piece of evidence considered in a defeasible reasoning process. Typically an argument is built upon a set of assumptions or premises, a method of reasoning and a conclusion.

Undermining attack: a type of conflict in which an argument is attacked on one of its premises by another argument whose conclusion negates that premise.

Rebutting attack: a type of conflict that occurs when an argument negates the conclusions of another argument.

Undercutting attack: a type of conflict that occurs when an argument uses a defeasible inference rule that is attacked by another argument arguing that there is a special case that does not allow the application of the rule itself.

Semantics: a formal criterion to determine which arguments of an argumentation graph can be accepted.

\section{State-of-the-Art: Defeasible Argumentation Theory}

\subsection{Defeasible Reasoning}

The capability of deriving defeasible conclusions with partial information is an important aspect of modern medical systems. In order to achieve such a capability, humans routinely resort to the so-called default knowledge, a main feature of which is that it can be used in a reasoning process even if the preconditions for its application are only partially known. These preconditions, whose truth is not explicitly verified, are assumed to hold defeasibly, that means in the absence of explicit information to the contrary. In the event that new information becomes 
available and the falsity of such preconditions can be deduced, then the conclusions derived from the application of the default knowledge have to be retracted. This type of reasoning is known as defeasible reasoning [4]. Default knowledge is represented by using defaults that are specific inference rules. These are expressions of the form: $p(x): j_{1}(x), \ldots, j_{n}(x) \longrightarrow c(x)$ where $p(x)$ is the prerequisite of the default, $j(x)$ is the justification and $c(x)$ is the consequent. If $p(x)$ is known and if $j(x)$ is consistent with what is known, then $c(x)$ can be defeasibly deduced. In other words, if it is believed that the prerequisite is true, and each of the $n$ conditions (justifications) can be assumed since they are consistent with current beliefs, then this leads to believe the truth of the conclusion. Defeasible reasoning, unlike standard deductive reasoning, is non-monotonic. Intuitively this means that adding new premises may lead to removing, rather than adding new conclusions. More specifically, if the conclusion $p$ follows from a set of premises $A$ (denoted as $A \vdash p$ ), in standard monotonic reasoning it also holds that $A, B \vdash p$ namely $t$, if and only if any additional set of premises $B$ is added to $A$, the conclusion $p$ is still valid. This property is called monotonicity: conclusions are not affected by new evidence hence the set of conclusions monotonically increases. This is not the case in real life in general and in medicine, health care in particular where reasoning is often non-monotonic: conclusions can be retracted when new evidence is available. Consider the following example [5]:

- X has undergone breast cancer surgery and subsequently radiotherapy.

- Radiotherapy minimises the risk of cancer recurrence, so possibly

- X has a low risk of breast cancer recurrence.

If in addition to the fact that $X$ has undergone cancer surgery and subsequently radiotherapy, it is found out that

\section{- X had a cancer with high degree of lymph node involvement,}

then the conclusion that $X$ has a low risk of cancer recurrence has to be retracted, as a special exception has been raised.

Non-monotonic logic relies on the idea that the pieces of knowledge employed in a reasoning activity such as $X$ has a low risk of cancer recurrence may admit exceptions and it is impossible to include a full list of exceptions within the reasoning rules [4]. In these cases, the premise of a certain rule is only partially specified and a conclusion can be derived from the premises, assuming that no exception occurs, that means that all the implicit premises of the rule are satisfied. In the case where an exception subsequently arises then the derived conclusion has to be retracted. The basic idea of non-monotonic inferences is that, when more information is obtained, some previously accepted inference may no longer hold. Defeasible reasoning has increasingly gained attention in the medical sector because it supports reasoning over partial, incomplete and dynamic evidence and knowledge, where several exceptions can arise according to various circumstances. Argumentation theory (AT), an important sub-field of artificial intelligence (AI), provides state-of-the-art computational models of defeasible reasoning (DR). 


\subsection{Argumentation Theory}

Argumentation theory (AT), often referred to as argumentation, is a multidisciplinary research subject ranging from law to philosophy and linguistic, with aspects borrowed from psychology and sociology [6,7]. AT has gained interest in artificial intelligence as it provides the basis for computational models inspired by the way humans reason [8]. These models have extended classical reasoning approaches, based on deductive logic, that were proving increasingly inadequate for problems requiring non-monotonic reasoning and explanatory reasoning not available in standard non-monotonic logics [9]. AT focuses on how pieces of evidence, seen as arguments, can be represented, supported or discarded in a defeasible reasoning process, and it investigates formal approaches to assess the validity of the conclusions inferred [6]. AT has been employed for tasks like practical reasoning, decision support, dialogue and negotiation [6,10-12] as well as for knowledge representation [13,14]. It differs from many traditional monolithic non-monotonic logics because it envisages a modular and intuitive process, supporting the explanation of each reasoning step, making the reasoning and inference processes more explanatory.

In a nutshell, argumentation deals with the interactions between possibly conflicting arguments, arising when different parties, participants or artificial agents argue for and against some conclusions or when different pieces of evidence, even conflicting, are available [12]. Arguments can be regarded as 'tentative proofs for propositions' [15] in a logical language whose axioms represent premises in the domain under consideration. In general, the premises are not consistent because they may lead to incompatible conclusions. These conflicts may arise either during the defeasible reasoning activity of a single human/agent or in the context of a dialogue between multiple humans or artificial agents. These modes are referred to as monological and dialogical argumentation, respectively. Accordingly, monological models [16] focus on the internal structure of an argument, meaning its components (like premises, rules, conclusions) and their relations. Dialogical models focus instead on argument conflicts and their resolution and typically regard arguments as monolithic entities, whose internal structure is abstracted away as far as the conflict resolution process is concerned. Roughly speaking, monological models concern the production and construction of arguments while dialogical models concern management of their conflicts, that means the actual arguing process. A third classification of models, referred to as rhetorical models, has also been proposed (Table 1) in which neither the monological nor the dialogical structure is considered [16]. Here, the rhetorical nature of arguments is stressed. More specifically, the audience's perception of arguments and how they can be employed as a means of persuasion is taken into account $[17,18]$.

In the literature of argumentation, models belonging to one category difficultly belong to the other categories. For instance, dialogical models do not address the internal representation of an argument and do not consider their perception by an audience. However, according to [16], in order to design intelligent systems that incorporate powerful argumentative capabilities, the micro- 
Table 1. Classification of argumentation models

\begin{tabular}{l|l|l|l}
\hline & Monological & Dialogical & Rhetorical \\
\hline Structure & Micro & Macro & Persuasive \\
\hline Foundation & $\begin{array}{l}\text { Arguments as tentative } \\
\text { proofs }\end{array}$ & Defeasible reasoning & $\begin{array}{l}\text { Audience's perception } \\
\text { of arguments }\end{array}$ \\
\hline Linkage & $\begin{array}{l}\text { Connecting a set of } \\
\text { premises to a claim at } \\
\text { the level of argument }\end{array}$ & $\begin{array}{l}\text { Connecting a set of } \\
\text { arguments in a } \\
\text { dialogical structure }\end{array}$ & $\begin{array}{l}\text { Connecting arguments } \\
\text { in a persuasive way }\end{array}$ \\
\hline
\end{tabular}

structure of an argument, its relation with other arguments as well as the rhetorical structure should be addressed. The internal representation of an argument should clearly relate premises to conclusions, and at an external level, the argument should be considered within the set of the other arguments it interacts with. Eventually, the perception by an audience is important because in real life implementations, arguments are built to achieve predefined objectives, according to the participating agents' believes. The general idea is that argumentation systems formalise non-monotonic reasoning as the internal construction of arguments (micro-structure) as well as their comparisons for and against certain conclusions (macro-structure). The construction of arguments, based on a theory, is monotonic that means an argument remains the same even if the theory is expanded with new information. Non-monotonicity is expressed in terms of interaction between conflicting arguments. This is because the additional information may generate stronger arguments that in turn defeat previous arguments.

Argumentation systems and the notion of an argument are typically constructed upon an underlying logical language and around an associated notion of logical consequence. As mentioned before, this notion of consequence is monotonic. New information can not invalidate existing arguments as constructed, but can only be responsible for the generation of new counterarguments. Some argument-based applications assume a particular and well-defined logic whereas other leave the underlying logic part of the context of application or even totally undefined. In the case the logic is left unspecified, the system can be instantiated with different alternative logics, thus they are often referred to as frameworks rather then systems. Beside the chosen underlying language, argumentation systems are generally built upon five layers [19] (Fig. 2):

1. definition of the internal structure of arguments

2. definition of conflicts between arguments

3. evaluation of conflicts and definition of valid attacks

4. definition of the dialectical status of arguments

5. accrual of acceptable arguments 
1) structure of arguments

2) conflicts of arguments

Translation of knowledge-base into interactive defeasible arguments

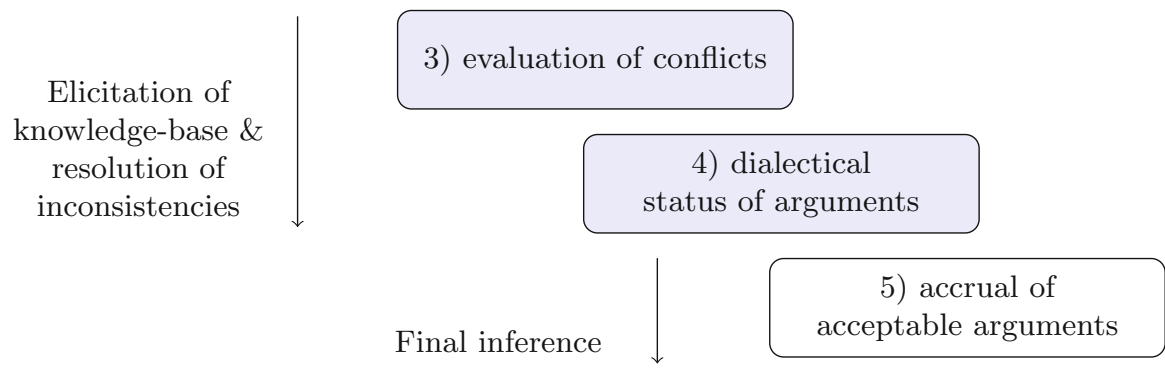

Fig. 2. Five layers upon which argumentation systems are generally built

\subsection{Layer 1: Definition of the Internal Structure of Arguments}

The internal representation of arguments is addressed by monological models. Often an argument is internally represented with a set of premises $\left(P_{1}, P_{2}, \ldots, P_{n}\right)$, and a conclusion $(C)$ follows from them with the application of some rule $(\rightarrow)$.

$$
\text { Argument : } P_{1}, P_{2}, \ldots, P_{n} \rightarrow C
$$

Many argumentation systems do not make any distinction between premises. However, arguments actually used in human reasoning may follow a more articulated structure where different premises play different roles, as in the argument model first introduced by Toulmin [20] composed of six parts (Fig. 3).

- Claim (C): an assertion/claim (conclusion) potentially controversial;

- Data (D): statements specifying facts/beliefs previously established related to a situation in which the claim is made;

- Warrant $(W)$ : statement that justifies the derivation of conclusion from data;

- Backing (B): a set of information that ensures the trustworthiness of a warrant. It is the grounds underlying the reason. A backing is invoked when the warrant is challenged;

- Qualifier $(Q)$ : a statement that expresses the degree of certainty associated with the claim;

- Rebuttal (R): a statement introducing a situation in which the conclusion might be defeated.

Toulmin's model plays a significant role in highlighting the elements that form a natural argument, providing a useful basis for knowledge representation. Another well-known monological paradigm has been proposed by Reed and Walton to model the notion of arguments as product [21,22]. It is based upon 
Fact (D)

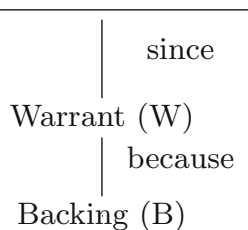

So (probably) (Q) Conclusion (C)<smiles></smiles>

Fig. 3. An illustration of the Toulmin's argument representation

the notion of an argumentation scheme and it is useful for identifying and evaluating a variety of argumentation structures in everyday discourse [16]. These argumentation schemes are aimed at capturing common stereotypical patterns of reasoning that are non-monotonic and defeasible in nature [13]. Consider the example presented in [8] in which two parts, A and B, are discussing chemotherapy, and that $\mathrm{B}$ is not in favour of it because $\mathrm{P}$ thinks that it has a high emotional impact on the person due to the risk of alopecia phenomenon and should be discouraged. B's argument is:

Argument: Dr. B (expert in psychology) says that chemotherapy affects the emotional state of the patient

It appears that B's argument is implicitly an appeal to expert opinion. In addition, it is evidently an instance of argument from consequences. These two schemes can be used by B to build a point of view. B is claiming that negatively affecting the emotional state is a bad consequence of an action. The argument is based upon the assumption that, since the bad outcome is a consequence of chemotherapy, therefore chemotherapy should not be applied. This can be represented by the following argumentation chain:

- Dr. B., an expert psychologist, says that chemotherapy negatively affects emotional state, because he has knowledge of patients emotions;

- chemotherapy negatively affects emotional state;

- negatively affecting the emotional state is a bad thing;

- anything that leads to bad consequences is a bad practice;

- chemotherapy is a bad practice.

Walton identified 25 different argumentation schemes, each including a set of critical questions such as:

'is the expert $E$ in a position to know about the proposition P?'

Critical questions provide a sort of checklist about the validity conditions for the application of a specific argument scheme. Intuitively, critical questions make the defeasibility of argument schemes explicit and indicate some canonical ways to build the relevant counterarguments. For further information on monological approaches to argumentation, readers can refer to [16]. The Toulmin's model [20] as well as the Reed and Walton's approach [21,22] do not specify the way different argument structures can be aggregated nor how they can interact or conflict in the dynamics of an argumentation process. 


\subsection{Layer 2: Definition of the Conflicts Between Arguments}

Monological models, aimed at representing the internal structure of arguments are complemented by dialogical models, focused on the relationships between arguments and, in particular, their conflicts. The latter investigates the issue of invalid arguments that appear to be valid (fallacious arguments). Conflicts, often referred to attacks or defeats, and sometimes with slightly different meanings, are the key notions in argumentation theory. In the AT literature several kinds of conflicts have been considered. Here the classification proposed in [23] is stressed. This encompasses three classes of conflicts (Figs. 4, 5, and 6):

- undermining attack: occurs when an argument is attacked on one of its premises by another whose conclusion negates that premise;

- rebutting attack occurs when an argument negates the conclusions of another;

- undercutting attack occurs when an argument uses a defeasible inference rule and is attacked by arguing that there is a special case that does not allow the application of the rule itself [24].

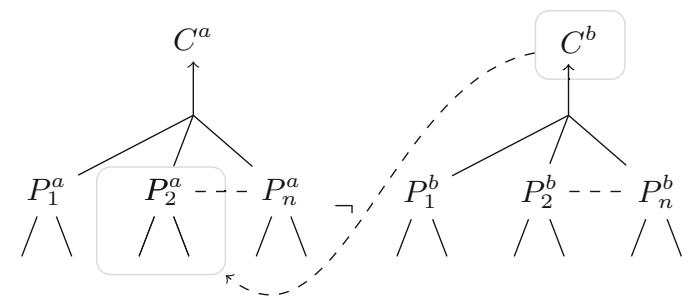

A: 'alcohol consumption is low according

B: the alcohol level from a blood test is to $X$ so $X$ has a low risk of recurrence' high so $X$ has a high alcohol consumption

Fig. 4. Undermining attack: $A$ is undermined by $B$

\subsection{Layer 3: Evaluation of Conflicts and Definition of Valid Attacks}

Conflict between arguments, although an important notion, does not embody any approach for the determination of the success of an attack, from one argument to its target. Generally an attack, sometimes referred to as 'defeat', has a form of a binary relation between two arguments. Some authors distinguish a relation in a weak form (attacking another argument and not weaker) or in a strong form (attacking another argument and stronger) [25]. The former is generally referred to as 'defeat' whereas the latter as 'strict defeat' [23]. Defeat relations are determined in various ways, influenced by the domain of application and are usually defeasible. For example, in those domains where observations are important, defeats might depend on the reliability of tests or the expertise of the observers. Evaluating an attack can occur through: 


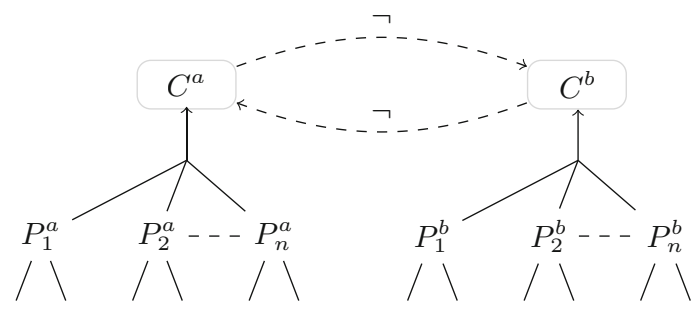

A: ' radiotherapy minimises risk so $X$ has a low risk of breast cancer recurrence
$\mathrm{B}$ : ' $X$ is an old patient, the strongest risk for breast cancer is age, so the risk of recurrence is high'.

Fig. 5. Rebutting attack: A is rebutted by B and viceversa
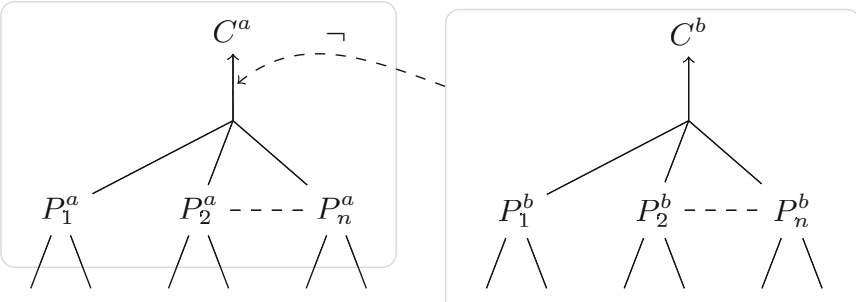

A: 'radiotherapy minimises the risk of recurrence so $X$ has a low risk of breast cancer recurrence'
B: 'paper $Z$ demonstrated that radiotherapy failed several times in curing breast cancer so it is not always an effective method to reduce recurrence'.

Fig. 6. Undercutting attack: $A$ is undercut by B

- the notion of preferentiality of arguments or strength of arguments

- the notion of preferentiality of attacks or strength of an attack relation.

Strength of Arguments. To establish whether an attack can be considered a successful defeat, a trend in AT is devoted to the consideration of the strength of arguments. In this respect a key concept is represented by the inequality of the strength of arguments that has to be accounted for in the computation of sets of arguments and counterarguments [26]. Several works have adopted the notion of preferentiality among arguments [27]. For example, in [28,29], the authors formalised the role of preferences and if an arguments $X$ undercuts another argument $Y$, then $X$ is a successful attack (defeat) if $Y$ is not stronger than $X$. Other approaches adopt preferentiality at a more abstract level. For instance, in the Preference-based Argumentation Framework (PAF) proposed by [30], an attack from $X$ to $Y$ is successful only if $Y$ is not preferred to $X$. [31] proposed 
a Value-based argumentation framework (VAF) in which an attack from $X$ to $Y$ is successful only if the value promoted by $X$ is ranked higher or equal than the valued promoted by $Y$, in accordance to a given ordering on values. Figure 7 illustrates these various scenarios of preferentiality, given an attack set and the resulting defeat (successful attack) set.

Starting attack set:

$$
0.5
$$

$\longleftarrow$ strength of arguments

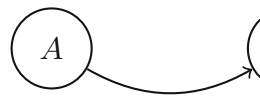

red

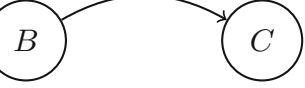

blue

blue

$\longleftarrow$ arguments

Preference of arguments: $[B>A>C$

Rank of values: $[$ red $>$ blue $]$

Resulting successful attack set:

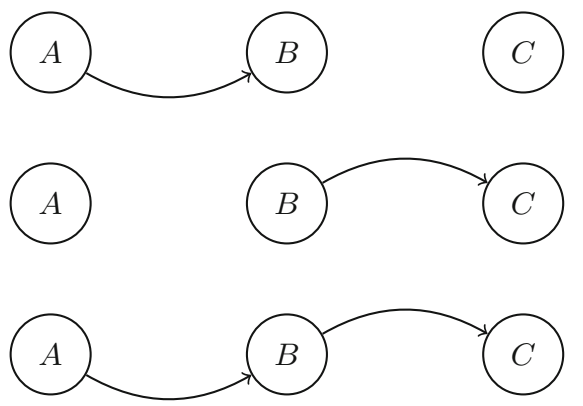

according to $[28]$ :

Pollock's defeasible reasoning

according to [30]:

preference-based relation (PAF)

according to [31]:

value-based argumentation (VAF)

Fig. 7. Implementations of preferentiality between arguments

The information necessary to decide whether an attack between two arguments is successful is often assumed to be pre-specified, and implemented as an ordering of values or a given partial preference. However, according to [27], the information related to preferentiality of arguments might be contradictory, as the preferences may vary depending on the context and on different subjects who can assign different strengths, to different arguments, employing different criteria. This led the author to propose the concept of meta-level argument: a simple node in a graph of nodes where preferentiality is abstractly defined, by creating a new attack relation that comes from a preference argument. Metalevel arguments allow no commitment regarding the definition of the preferences of arguments, rendering the reasoning process simpler. To model the preference relation among arguments, the notion of fuzziness has been used in [32] where a fuzzy preference argumentation framework (FPAF) has been proposed. Here, 


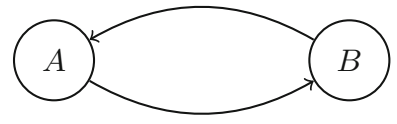

Preference list: $[A>B]$

(a) Standard preferentiality

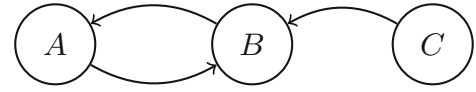

Preference argument C

(b) Meta-level argument

Fig. 8. Standard preferentiality and meta-level arguments

a value $X$ attached to a preference relation between two arguments $A, B$ corresponds to the degree of credibility by which $A$ is strictly preferred to $B$. To clarify the above notions, consider the example of Fig. 8 where two arguments A, B, claiming two different conclusions rebut each other. Suppose the existence of a pre-defined preference list in which argument $\mathrm{A}$ is preferred to argument B (part a). According to Modgil [27], this situation can be expressed as in the graph (part b) where another meta-level argument $\mathrm{C}$ is added to the reasoning process, undercutting the inference link of argument B.

Strength of Attack Relations. Preferentiality, as reviewed so far, is implemented by assigning to arguments an importance value. This is usually predefined, in form of a full or partial priority list of available arguments, or in form of a numerical value attached to each of them, explicitly provided or implicitly derived from the strength of the rules used within the argument. In turn preferentiality allows to establish whether an attack can be considered successful, thus formalising a proper defeat relation, or considered a weak/false attack, thus being disregarded. As opposite to this approach, another branch of argumentation is devoted to associate weights to attack relations instead to arguments. In [26] the role of adding weights on the attack links between arguments has been investigated, introducing the notion of inconsistency budget. This quantifies the amount of inconsistency a designer of an argumentation system is willing to tolerate. With an inconsistency budget $\alpha$, the designer is open to disregard attacks up to a total weight $\alpha$. It turns out that, increasing this threshold, more solutions can be achieved progressively as less attack would be disregarded. As a consequence, this gives a preference order over solutions, and the solutions having a lower inconsistency budget are preferred. A similar recent approach that considers the strength of attacks is incorporated in [33] resulting in a variedstrength attacks argumentation framework (VSAAF). Here, each attack relation is assigned a type, and the framework is equipped with a partial ordering over the types. Let us consider the example of Fig. 9 where the type of attack from an argument $A$ to $B$ is $i$ and from $B$ to $C$ is $j$. Intuitively, depending on whether the type $j$ is higher, lower or equally ranked than the type $i$, different ranges of solutions are possible. Beside this relation of attack, another approach has been proposed [34] by employing the notion of fuzzy relations borrowed from Fuzzy 


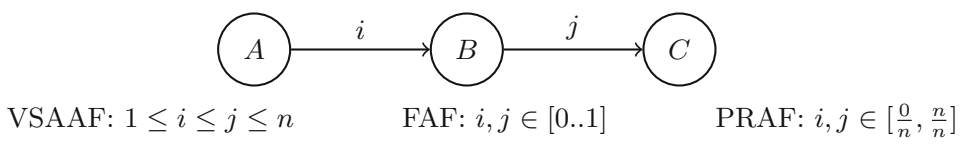

Fig. 9. Types of the strength of attack relations

Logic $[35,36]$. This approach allows the representation of the degree to which an argument attacks another one, creating a fuzzy argumentation framework (FAF).

Strength of arguments and defeat relations has been considered also in [37]. Here, probabilities are assigned both to arguments and defeats, introducing the notion of probability argumentation framework (PRAF). Probabilities refer to the likelihood of the existence of a specific argument or defeat relation, thus capturing the inherent uncertainties in the argumentation system. The idea is that all possible arguments neither definitely are disregarded nor they definitely exist: they have different chances of existing. In the approach proposed in [38] two fictitious people have to be confronted, endorsing respectively the roles of proponent and opponent of the argument. Situation of conflicts are subsequently analysed employing the paradigm of game theory [38].

\subsection{Layer 4: Definition of the Dialectical Status of Arguments}

Defeat relations, as per layer 3, focus on the relative strength of two individual arguments and do not tell yet what arguments can be seen as justifiable. The final state of each argument depends on the interaction with the others and a definition of their dialectical status is needed. Layer 4 of the multi-layer schema of Fig. 2 is aimed at determining the outcome of an argumentation system usually by splitting the set of arguments in two classes, those that support a certain decision/action and those that do not. Sometimes a further class can contain those arguments that leave the dispute in an undecided status. Multiple actions or decisions can be accounted for in a defeasible reasoning process, thus the number of classes can increase. Modern implementations for computing the dialectical status of arguments are usually built upon the theory of Dung [9] which, historically speaking, derives from other more practical and concrete works on argumentation such as $[24,39]$. Dung's abstract argumentation frameworks (AF) allow comparisons of different systems by translating them into his abstract format [39]. The underlying idea is that given a set of abstract arguments (the internal structure is not considered) and a set of defeat relations, a decision to determine which arguments can ultimately be accepted has to be taken. AF is a directed graph in which arguments are presented as nodes and the attacks as arrows (Fig. 10). Solely looking at an argument's defeaters to decide the status of an argument is not enough: it is also important to investigate whether the defeaters are defeated themselves. Generally, an argument $B$ defeats $A$ if and only if $B$ is a reason against $A$.

Given an AF, the issue is to decide which arguments should ultimately be accepted. In Fig. 10, $A$ is attacked by $B$, and apparently $A$ should not be accepted 


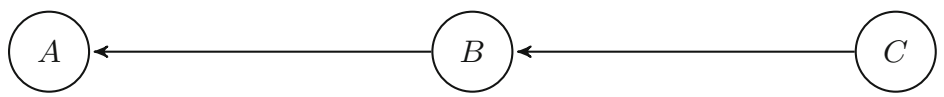

Fig. 10. Argument and reinstatement

since it has a counterargument. However, $B$ is itself attacked by $C$ that, in turn, is not attacked by anything, thus $C$ should be accepted. But if $C$ is accepted, then $B$ is ultimately rejected and does not form a reason against $A$ anymore. Therefore $A$ should be accepted as well. In this scenario it is said that $C$ reinstates $A$ and in order to determine which arguments of an $\mathrm{AF}$ can be accepted, a formal criterion is necessary. This criterion is known as acceptability semantics, and given an AF, it specifies zero or more extensions (sets of acceptable arguments) [40]. Using the labelling approach proposed in [41], each argument is either in, out or undec according to two conditions:

1. an argument is labelled in if, only if all its defeaters are labelled out, and 2. an argument is labelled out if, only if it has at least one defeater labelled in.

Informally speaking, an argument labelled in means it has been accepted, out means it has been rejected and undec means it can not be neither accepted nor rejected. In the AF of Fig. 10, for argument $C$ it holds that all its defeaters are labelled out (trivial as $C$ is not defeated by any argument), thus $C$ has to be labelled in. $B$ now has a defeater labelled in thus it has to be labelled out. For $A$, it holds that all its defeaters are labelled out, so it has to be labelled $i n$. As a consequence the resulting status of each argument is: $\operatorname{Lab}(A)=i n$, $\operatorname{Lab}(C)=$ in and $\operatorname{Lab}(B)=$ out. Thus, $A$ and $C$ can be accepted and argument $B$ has to be rejected. A set of arguments is called conflict-free if and only if it does not contain any argument $A$ and $B$ such that $A$ defeats $B$. A set of arguments Args is said to defend an argument $C$ if and only if each defeater of $C$ is defeated by an argument in Args. These basic notions drive the proposal of the complete semantics aimed at computing complete extensions [9]. The idea is that a complete labelling might be viewed as a subjective and reasonable point of view that a designer can consider with respect to which arguments are accepted, rejected or considered undecided. Each point of view can be certainly questioned by someone, but its internal inconsistency cannot be pointed out. The set of complete labellings can be seen as the reasonable positions available to a designer [41].

Complete semantics have an important property: more than one complete extension might exist. However, sometimes it is advantageous to take a skeptical approach, thus a semantics that is guaranteed to generate exactly one extension is the grounded semantics. The idea is to select the complete labelling Lab in which the set of $i n$-labelled arguments is minimal. The grounded extension coincides with the complete labelling in which in is minimised, out is minimised and undec is maximised and can be the empty set. In Fig. 10, the grounded extension is $\{A, C\}$. However, this skeptical approach might be replaced by a more credulous one, known as preferred semantics [9]. The idea is that, instead 
of maximising undec arguments, it maximises in arguments (and also out arguments). They are based on the notion of admissibility. A set of arguments is admissible if and only if it is conflict-free and defends at least itself. The empty set is admissible in every AF as it is conflict-free and trivially defends itself against each of its defeaters (none). For any AF, there exists at least one preferred extension. Every grounded and every preferred extension is a complete extension. In Fig. 10, the admissible sets are $\{C\},\{A, C\} .\{B\}$ and $\{A\}$ are not admissible as they do not defend themselves respectively against $C$ and $B$. Only one preferred extension exists: $\{A, C\}$. Grounded and preferred semantics have been conceived by Dung and firstly described in his topical work [9]. However, other semantics have been proposed such as the ideal semantics $[42,43]$, semistable [44], stage [45], non-admissibility based semantics [46] and CF2 semantics [47]. For further readings on argumentation semantics, the reader is referred to $[40,48]$.

\subsection{Layer 5: Accrual of Acceptable Arguments}

Multiple acceptable extensions of arguments may be computed from the previous layer coinciding with possible consistent points of view that can be considered for describing the knowledge being modelled and thus employed for decisionmaking and defeasible inference. However, sometimes for practical purposes, as in the medical domain, a single decision must be takes or a single action must be performed. Thus a fifth layer is sometimes added to the argumentative schema aimed at extracting the most credible or consistent point of view for informing such a decision or action. It includes a strategy for computing, for instance, a degree of credibility of each extension that can be used for purposes of comparison. The most credible can be eventually selected and employed to support decision-making. Various strategies have been proposed in the literature for selecting such an extension $[49,50]$. These include the consideration of the strength of arguments, or a preference list among them defined in layer 3 . Alternatively, the extension with higher cardinality can be considered, that is the larger conflict-free set of arguments. In the literature of argumentation and defeasible reasoning, this layer is probably the less developed and further works should be carried out.

\section{Application Areas}

The previously described five layers (Fig. 2) give an overall idea of the main components that are usually considered in an argumentative process, and are strictly connected. The first layer deals with monological argumentation while the other layers with dialogical argumentation. Some of these layers can be neglected or merged together. For example, when the strength of arguments or attack relations is not considered, layer 3 can be discarded. Also, the strength of arguments and their preferentiality may be considered in the 5th layer and not only in the 3rd layer. The literature of defeasible reasoning and its theoretical works is vast 
in the logic and artificial intelligence communities. Readers can refer to [16] for a taxonomy of argument-based models and to [51] for a review of defeasible reasoning implementations. In this section, applications of defeasible reasoning and argumentation in medicine and health-care are described. Argumentation was adopted in the context of the Aspic project [52]: a general model for argumentation services. The goal was to develop a theoretical framework for inference, decision-making, dialogue and learning that could be used, for example, in the identification of patients treatment options given multiple and conflicting pieces of evidence. An application of this framework includes a multi-agent scenario where three agents collaborate exchanging pros and cons of alternative interventions and diagnoses towards settling on a justifiable treatment for a patient with chest pain [53]. Aspic has been also used as method for genetic counselling aimed at providing patients and clinicians with an aid for customising, evaluating, visualising and communicating care plans. Another application concerned a simulation where eight cancer genetic counsellors participated in an experiment in which they had to counsel a woman carrying a risk-increasing gene mutation. Information was visually displayed in an organised-fashion, in the form of structured arguments. These arguments helped counsellors enhancing their discussion with the patient and explaining the options available for mitigating the risk of cancer [54]. In the Aspic project, arguments are constructed from a knowledgebase of facts, internally modelled with strict and defeasible inference rules. These rules are composed by a set of premises supporting a claim and an argument can embed different rules organised as a tree. Each argument has a numerical degree of belief attached ( 1 for strict arguments and a partial degree, less than 1, for defeasible arguments), and this can be computed employing different principles. These include the 'weakest link' principle in which the minimum of the strength of an argument's premises and its links is computed, or the 'last link' principle in which the maximum strength of an argument's links, with no accrual of reasons is considered [55]. Once arguments are defined, the Aspic framework allows the explication of a set of attack relations between them, always according to the knowledge-base of facts. Dung's calculus of opposition [9] is employed to compute a dialectical (justification) status of arguments. Eventually, from the claim of the justified arguments, a final inference is drawn, this being usually a decision, a diagnosis or a treatment recommendation.

Argumentation has been used for medical group-decision support [56]. In this context, expert clinicians participated in a group discussion to decide on the best treatment for a given patient or case. A web-prototype to build arguments was presented to a group of oncologists who were asked to discuss on treatment therapies for patients having cancer in the head-to-neck region. Arguments were modelled as natural language propositions constructed upon a particular piece of evidence, acquired from the literature, and linked to a particular treatment choice. Each argument was also accompanied by a value indicating the strength of the underlying evidence. A machinery that extended Dung's calculus of opposition [9] was proposed, followed by a preference-based accrual of arguments [56]. Further research studies adopted the Aspic framework in the context of 
consensus on explanations and it focused on understanding how two clinicians, with a disagreement in relation to an anomalous patient's response to treatment, exchanged arguments in order to arrive at a consensus [57]. Gorogiannis et al. employed argumentation for investigating treatment efficacy and their work was motivated by the fact that, although there was a rapidly-growing dataset of trial results, this dataset was inconsistent, incomplete and required a significant effort to be sensibly aggregated for the inference of a single correct decision [58]. The authors proposed an argument-based framework to analyse the available knowledge and present the different possible results. In this framework, the monological structure of arguments was modelled as a triple $\langle A, B, C\rangle$ with $A$ the set of evidence from a clinical trail, $B$ an inference rule that linked evidence to a claim $C$. The claim was a comparison between the outcomes of two generic treatments $t 1$ and $t 2$ (only two-arm comparisons were treated) that can be either $t 1>t 2$ ( $t 1$ is statistically superior to $t 2), t 2<t 1$ (viceversa) or $t 1 \sim t 2$ (no statistical difference). Regarding the dialogical structure, arguments (clinical tests) conflicted with each other if they entailed contradicting claims and contradictions were resolved with the Dung's calculus of opposition. This framework was extended in [59] by allowing the expression of preferences among arguments and by employing descriptive logic to further specify their monological structure. In this extension, authors performed a case study on ovarian cancer data showing how the introduction of the dialogical Dung's calculus of opposition could support the selection of relevant/undisputed clinical evidence in a large and fragmented dataset of cases.

Argumentation has been employed for predicting the recurrence of breast cancer in patients who have undergone a surgery [60]. In this circumstance, the knowledge-base of a cancer expert has been translated into arguments with premises supporting either recurrence or non-recurrence of cancer. This monological structure has been subsequently extended adding conflicts among arguments organised dialogically, always according to the expert's knowledge-base. In turn, they were evaluated with the Dung's calculus of opposition. A strategy based on the largest cardinality was implemented for selecting the most credible preferred extension, and thus recommending a justifiable outcome (recurrence or non-recurrence). [61] describes an application of argumentation to the field of organ transplant called Carrel+. Human-organ is a decision-making process that often illustrates conflicts among medical experts: what may be sufficient for one doctor to discard an organ may not be for another one. This application allows doctors to express their arguments about the viability of an organ and employs monological argumentation techniques, namely argumentation schemes [20] and critical questions [21] to combine arguments, to identify inconsistencies and to propose a valid solution considering their relative strength as well as the available evidence about the organ and the donor. Other ways to elaborate and construct arguments exist and they differ because of the variability of their monological structure. For instance, [58,59] are different from [61-63]. In the former studies, arguments are built directly from clinical trial results with a uniform structure that makes the approach less domain-dependent and scalable to 
large-volume data. In the latter works, arguments are hand-crafted and ad-hoc constructs built by relying on domain specific expertise and therefore they have a variable internal structure.

Ultimately, [64] is probably the most complete work applying argumentation to medical decision support. This work is closely adhering to the 5-layer schema previously introduced (Sect.3). First, the available evidence, collected from experts or literature, is converted into a monological argument structured as an inference rule. Second, a medical expert can set up preference relations by assigning a weight to each arguments (argument $\mathrm{A}$ can be preferred to $\mathrm{B}$ because, despite having comparable effects, A has fewer side effects than B). Third, meta-arguments can be built about the quality of arguments created in the first stage (an argument based on a non-randomised small sample is weaker than another based on the evidence collected on a large randomised sample). Forth, the dialogical structure is arranged in a Dung style argumentation graph and an argumentation semantics is used for computing their dialectical and acceptability status from which consistent conclusions can be suggested to the decision makers. The study proposes several case studies: diagnosis of glaucoma, treatment of hypertension and treatments of pre-eclampsia.

In summary, Table 2 gives a panoramic of the contributions reviewed so far, classified according to the 5-layer schema introduced in Fig. 2 The aim is at providing the reader with a high-level snapshot describing the current effort devoted towards producing argument-based systems in medicine and healthcare.

\subsection{Features of Argumentation}

Theoretically, argumentation and defeasible reasoning have a set of features that are generally appealing and specifically interesting for clinicians and practitioners in the field of medicine and health-care $[7,60]$.

- Inconsistency/incompleteness: argumentation provides a methodology for reasoning on available evidence, even if this evidence is partial and inconsistent as it often happens in medicine and health-care;

- Expertise/uncertainty: argumentation captures expertise in an organised fashion, employing the notion of arguments and it can handle vagueness and the uncertainty associated with clinical evidence;

- Intuitiveness: argumentation is close to the way humans reason. Vague knowledge bases can be structured as arguments built with familiar linguistic terms, which is extremely appealing for clinicians;

- Explainability: argumentation leads to explanatory reasoning thanks to its incremental, modular way of reasoning with available evidence. It provides approaches for computing the justification status of arguments, allowing the final decision of a reasoning process to be better explained;

- Dataset independency: argumentation does not require a complete dataset and it may be useful for emerging knowledge, where quantitative evidence has not yet been gathered or is limited; 
Table 2. Argument-based systems in medicine and health-care: applications

\begin{tabular}{|c|c|c|c|c|c|c|}
\hline Ref & Domain & Layer 1 & Layer 2 & Layer 3 & Layer 4 & Layer 5 \\
\hline$[52,53]$ & $\begin{array}{l}\text { argumentation } \\
\text { services, } \\
\text { patient } \\
\text { treatment } \\
\text { options }\end{array}$ & $\begin{array}{l}\text { a tree of } \\
\text { premises } \rightarrow \text { claim }+ \\
\text { degree of belief }\end{array}$ & $\begin{array}{l}\text { abstract } \\
\text { attacks }\end{array}$ & $\begin{array}{l}\text { degree of } \\
\text { belief }\end{array}$ & dung & $\mathrm{n} / \mathrm{a}$ \\
\hline$[54]$ & $\begin{array}{l}\text { genetic } \\
\text { consueling, } \\
\text { care plans }\end{array}$ & Toulmin & $\mathrm{n} / \mathrm{a}$ & $\mathrm{n} / \mathrm{a}$ & $\mathrm{n} / \mathrm{a}$ & $\mathrm{n} / \mathrm{a}$ \\
\hline$[56]$ & $\begin{array}{l}\text { group- } \\
\text { decision } \\
\text { support }\end{array}$ & $\begin{array}{l}\text { natural language } \\
\text { propositions }+ \\
\text { strength of evidence }\end{array}$ & $\begin{array}{l}\text { abstract } \\
\text { attacks }\end{array}$ & $\begin{array}{l}\text { preference } \\
\text { list }\end{array}$ & dung + & $\begin{array}{l}\text { preference- } \\
\text { based }\end{array}$ \\
\hline$[57]$ & $\begin{array}{l}\text { consensus, } \\
\text { explanations }\end{array}$ & $\begin{array}{l}\text { a tree of } \\
\text { premises } \rightarrow \text { claim }+ \\
\text { degree of belief }\end{array}$ & $\begin{array}{l}\text { abstract } \\
\text { attacks }\end{array}$ & $\begin{array}{l}\text { degree of } \\
\text { belief }\end{array}$ & dung & $\mathrm{n} / \mathrm{a}$ \\
\hline$[60]$ & $\begin{array}{l}\text { cancer } \\
\text { prediction }\end{array}$ & premises $\rightarrow$ claim & $\begin{array}{l}\text { abstract } \\
\text { attacks }\end{array}$ & $\mathrm{n} / \mathrm{a}$ & dung & $\begin{array}{l}\text { extension } \\
\text { cardinality }\end{array}$ \\
\hline$[58]$ & $\begin{array}{l}\text { treatment } \\
\text { efficacy }\end{array}$ & premises $\rightarrow$ claim & $\begin{array}{l}\text { abstract } \\
\text { attacks }\end{array}$ & $\begin{array}{l}\text { preference } \\
\text { list }\end{array}$ & dung & $\mathrm{n} / \mathrm{a}$ \\
\hline$[59]$ & $\begin{array}{l}\text { identification } \\
\text { of relevant } \\
\text { evidence }\end{array}$ & descriptive logic & $\begin{array}{l}\text { abstract } \\
\text { attacks }\end{array}$ & $\begin{array}{l}\text { preference } \\
\text { relation- } \\
\text { ships }\end{array}$ & $\begin{array}{l}\text { dialectical } \\
\text { tree }\end{array}$ & $\mathrm{n} / \mathrm{a}$ \\
\hline$[61]$ & $\begin{array}{l}\text { organ } \\
\text { transplant } \\
\text { confirmation }\end{array}$ & $\begin{array}{l}\text { argument schemes } \\
+ \text { critical questions }\end{array}$ & $\begin{array}{l}\text { abstract } \\
\text { attacks }\end{array}$ & $\begin{array}{l}\text { argument } \\
\text { strength }\end{array}$ & dung & $\mathrm{n} / \mathrm{a}$ \\
\hline$[62]$ & $\begin{array}{l}\text { breast cancer } \\
\text { care }\end{array}$ & $\begin{array}{l}\text { Evidence-based } \\
\text { guideline }\end{array}$ & $\mathrm{n} / \mathrm{a}$ & $\mathrm{n} / \mathrm{a}$ & $\mathrm{n} / \mathrm{a}$ & $\mathrm{n} / \mathrm{a}$ \\
\hline$[64]$ & $\begin{array}{l}\text { Treatments of } \\
\text { diseases }\end{array}$ & inference rule & $\begin{array}{l}\text { argument } \\
\text { strength }\end{array}$ & $\begin{array}{l}\text { meta } \\
\text { arguments }\end{array}$ & dung & $\begin{array}{l}\text { utility } \\
\text { theory }\end{array}$ \\
\hline
\end{tabular}

- Extensibility/updatability: argumentation is an extensible paradigm that allows a decision to be retracted in the light of new evidence. An argumentation system can be updated with new arguments when they become available;

- Knowledge-bases comparability: argumentation allows comparisons of different subjective knowledge-bases. Two clinicians might build their own argumentation frameworks, identify differences in the definition of their beliefs, expertise and intuitions as well as compare their inferential capacity;

- Consensus building: argumentation is a useful approach for decision-making and achieving consensus between contradicting perspectives of knowledge.

Although argumentation has a great potential for supporting decisionmaking, enhancing knowledge representation and performing defeasible inference in the light of fragmented, partial, vague and inconsistent knowledge, it has some limitations $[10,11]$. The aforementioned features are appealing at the theoretical level, however, there are more practical, open problems for applied research. The following section is aimed at describing these problems and present future challenges for enabling wide-spread application of argumentation at the more practical level. Readers can refer to [11] for a further discussion on the role of argumentation and argumentation-based applications in modern computing. 


\section{Open Problems and Challenges}

Bench-Capon and Dunne discussed limitations of argumentation in artificial intelligence and computer science [10], identifying a set of challenges concerning the widespread deployment of argumentation technology. These challenges are still valid and their resolution requires the union of theoretical work with more practical engineering work. Probably, the most important limitation concerns the adoption of argumentation methods and systems in practical fields, these including medicine and health care. However, other limitations exist:

- lack of engineering solutions for application/automation of argumentation

- lack of a strong link between argumentation and other formalisms for dealing with uncertainty;

- scalability of argument-based applications and their widespread;

- ambiguity of the communication protocols and language that can be used by artificial agents incorporating argumentative capabilities.

Firstly, as it often happens in real-world knowledge-engineering, pieces of knowledge are abundant, so the amount of arguments that can be built upon them. However, engineering and software tools for the monological representation of arguments are limited, despite advances in technology and interfaces. Diagrammatic representations of arguments have been proposed $[20,21,65]$ but their implementation in practice is still narrow. Human reasoning over graphical diagrams is fundamental to enable human experts to translate their knowledge bases and beliefs in a computable form employable for reasoning and inference. User-friendly interfaces are necessary for enabling human operators to link arguments together, for modelling their conflicts and for performing inference through the execution of acceptability semantics for the resolution of these conflicts. These bottlenecks must be addressed to support the deployment and adoption of argument-based applications. Advances in user-interface design and deployment as well as the availability of web-based tools (javascript) are valid candidates for tackling these bottlenecks.

Secondly, in order to facilitate the impact of argument-based applications in the arena of intelligent computer systems, a further challenge is the construction of a stronger link with other formalisms for dealing with uncertainty within the broader field of artificial intelligence. Examples include probability and Bayesian theories, Dempster-Shafer theory and Game Theory [38] for applications requiring the interaction of multiple parties, participants [56] or Fuzzy Sets and Logic $[32,34-36]$ for representation of vague knowledge. Further formalisms concerning the supporting of collaborative work/learning or decision-making [52] include Organisational Theory $[66,67]$ and Decision theory [68].

Thirdly, another important challenge refers to the scalability of applications of argumentation and their widespread. This means that in order to demonstrate the impact of argument-based technologies to knowledge representation and reasoning, several applications have to be deployed and tested in different disciplines such as education, medicine $[54,56,57,64]$, psychology, biology extending traditional fields of application such as artificial intelligence [10], computer science, 
philosophy, linguistic and human-computer Interaction [69-71]. The scalability challenge also refers to a more technical issue concerning the deployment of engineering systems that can easily scale, such as in [72], and let a great amount of parties, participants or artificial agents to be engaged in a large-scale argumentative process and enabling collective intelligence [73]. Assuming the above challenges can be resolved there is another important challenge referred to the development of the protocols for allowing artificial agents, incorporating argumentative capabilities, to communicate, argue and negotiate with each other in a distributed digital world $[11,74]$. Recent advances in the field of multi-agent systems might offer valid approaches to tackle this problem.

\section{Future Outlook}

This chapter has presented an overview of argumentation for knowledge representation, conflict resolution and defeasible reasoning, with an informal description of the multi-layer pattern usually adopted for implementing in practice such reasoning. A literature review of applications of argumentation showed how defeasible reasoning has been employed so far in the medical and health-care sectors. Advantages and features of argumentation have been proposed emphasising the benefits for defeasible inference under uncertainty. Open problems and challenges have been identified, these mainly referring to the practical applicability of argumentation rather than the development of new theoretical formalisms. The lack of user-friendly tools and procedures employable by humans to build arguments, connect them in a dialogical structure and enable defeasible reasoning in practice is the most important challenge for applied research. From a more theoretical perspective, future work should be focused on the integration of argumentation theory and machine learning as two different but complementary methods for enhancing knowledge representation and extraction, reasoning and classification with fragmented, partial and conflicting information [75]. This integration could be tackled through 3 points of interaction (Fig. 11, A, B, C).

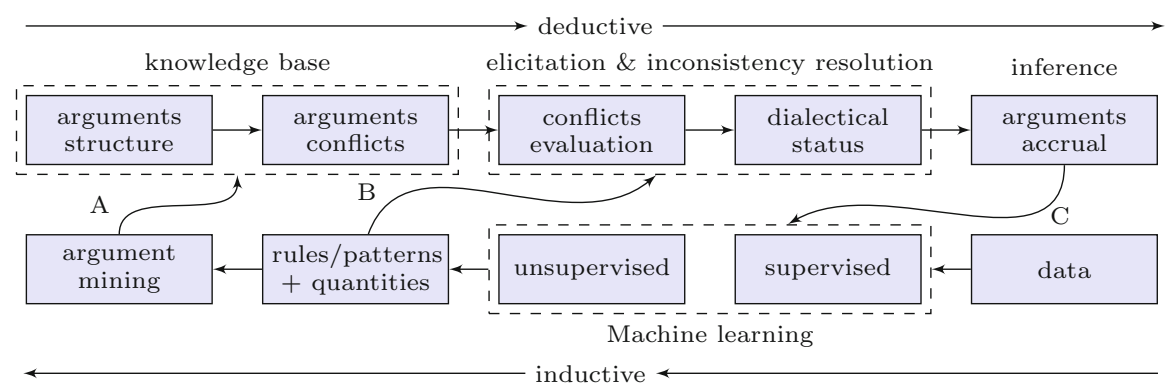

Fig. 11. Integration of machine learning and defeasible argumentation

On one hand, argumentation represents a theory and belief-driven deductive paradigm to reasoning over pieces of evidence, potentially conflicting and 
fragmented towards a final inference. On the other hand, machine learning, is a data-driven inductive paradigm aimed at exploring data, extracting patterns and building learning models from it so classification can be performed and predictions can be made. Through these models, new arguments can be built and incorporated in existing knowledge-bases thus enhancing reasoning processes (A) [76]. The rules and patterns emerged from unsupervised machine learning techniques and the predictions, probabilistic values available in supervised machine learning classifiers can support the resolution of potential inconsistencies in a defeasible reasoning process, providing it with numerical attributes useful for formalising preferences and deciding between conflicting arguments (B). Eventually, the inference produced by a defeasible reasoning process can provide machine learning with a useful theoretical background for identifying deceptive chains of inference that might lead to erroneous results (C). Hybrid models employing both the paradigms can benefit from the advantages of each approach and tackle their pitfalls and they are expected to perform better in term of representation, clustering of knowledge and in term of prediction and inference.

\section{References}

1. Holzinger, A.: Interactive machine learning for health informatics: when do we need the human-in-the-loop? Brain Inform. 3, 1-13 (2016)

2. Holzinger, A., Plass, M., Holzinger, K., Crişan, G.C., Pintea, C.-M., Palade, V.: Towards interactive Machine Learning (iML): applying ant colony algorithms to solve the traveling salesman problem with the human-in-the-loop approach. In: Buccafurri, F., Holzinger, A., Kieseberg, P., Tjoa, A.M., Weippl, E. (eds.) CDARES 2016. LNCS, vol. 9817, pp. 81-95. Springer, Heidelberg (2016). doi:10.1007/ 978-3-319-45507-5_6

3. Gomez, S.A., Chesnevar, C.I.: Integrating defeasible argumentation with fuzzy art neural network for pattern classification. J. Comp. Sci. Technol. 4(1), 45-51 (2004)

4. Baroni, P., Guida, G., Mussi, S.: Full nonmonotonicity: a new perspective in defeasible reasoning. In: ESIT 1997, European Symposium on Intelligent Techniques, pp. 58-62 (1997)

5. Longo, L., Dondio, P.: Defeasible reasoning and argument-based medical systems: an informal overview. In: 27th International Symposium on Computer-Based Medical Systems, pp. 376-381. IEEE, New York (2014)

6. Toni, F.: Argumentative agents. In: The Multiconference on Computer Science and Information Technology, pp. 223-229 (2010)

7. Longo, L., Kane, B., Hederman, L.: Argumentation theory in health care. In: Proceedings of CBMS 2012, The 25th IEEE International Symposium on ComputerBased Medical Systems, June 20-22, Rome, Italy, pp. 1-6 (2012)

8. Longo, L.: Formalising Human Mental Workload as a Defeasible Computational Concept. PhD thesis, Trinity College Dublin (2014)

9. Dung, P.M.: On the acceptability of arguments and its fundamental role in nonmonotonic reasoning, logic programming and n-person games. Artif. Intell. 77(2), 321-358 (1995)

10. Bench-Capon, T.J., Dunne, P.E.: Argumentation in artificial intelligence. Artif. Intell. 171(10-15), 619-641 (2007) 
11. Rahwan, I., McBurney, P.: Argumentation technology (guest editors). IEEE Intell. Syst. 22(6), 21-23 (2007)

12. Matt, P.A., Morgem, M., Toni, F.: Combining statistics and arguments to compute trust. In: International Conference on Autonomous Agents and Multiagent Systems (2010)

13. Dondio, P., Longo, L.: Computing trust as a form of presumptive reasoning. In: 2014 IEEE/WIC/ACM International Joint Conferences on Web Intelligence (WI) and Intelligent Agent Technologies (IAT), vol. I, Warsaw, Poland, August 11-14, pp. 274-281 (2014)

14. Longo, L.: A defeasible reasoning framework for human mental workload representation and assessment. Behav. Inf. Technol. 34(8), 758-786 (2015)

15. Krause, P., Ambler, S., Elvang-Gransson, M., Fox, J.: A logic of argumentation for reasoning under uncertainty. Comput. Intell. 11(1), 113-131 (1995)

16. Bentahar, J., Moulin, B., Blanger, M.: A taxonomy of argumentation models used for knowledge representation. Artif. Intell. Rev. 33(3), 211-259 (2010)

17. Grasso, F.: Towards a framework for rhetorical argumentation. In: Proceedings of the 6th Workshop on the Semantics and Pragmatics of Dialogue, pp. 53-60 (2002)

18. Pasquier, P., Rahwanm, I., Dignum, F., Sonenberg, L.: Argumentation and persuasion in the cognitive coherence theory. In: The 1st International Conference on Computational Models of Argument, pp. 223-234 (2006)

19. Prakken, H., Vreeswijk, G.: Logics for defeasible argumentation. In: Gabbay, D.M., Guenthner, F. (eds.) Handbook of Philosophical Logic, vol. 4, pp. 219-318. Springer, Heidelberg (2002)

20. Toulmin, S.: The use of argument. Cambridge University Press, Cambridge (1958)

21. Walton, D.: Argumentation Schemes for Presumptive Reasoning (Studies in Argumentation Theory). Lawrence Erlbaum Associates, Inc., Hillsdale (1996)

22. Reed, C., Walton, D.: Argumentation schemes in argument-as-process and argument-as-product. In: Proceedings of the Conference Celebrating Informal Logic, vol. 25 (2003)

23. Prakken, H.: An abstract framework for argumentation with structured arguments. Argument and Comput. 1(2), 93-124 (2011)

24. Pollock, J.L.: Justification and defeat. Artif. Intell. 67(2), 377-407 (1994)

25. Martínez, D.C., García, A., Simari, G.R.: Strong and weak forms of abstract argument defense. In: Proceedings of the 2008 Conference on Computational Models of Argument: Proceedings of COMMA 2008, pp. 216-227. IOS Press (2008)

26. Dunne, P.E., Hunter, A., McBurney, P., Parsons, S., Wooldridge, M.: Weighted argument systems: basic definitions, algorithms, and complexity results. Artif. Intell. 175(2), 457-486 (2011)

27. Modgil, S.: Reasoning about preferences in argumentation frameworks. Artif. Intell. 173(9-10), 901-934 (2009)

28. Pollock, J.L.: Defeasible reasoning. Cognitive Sci. 11(4), 481-518 (1987)

29. Prakken, H., Sartor, G.: Argument-based extended logic programming with defeasible priorities. J. Appl. Non-Class. Logics 7, 25-75 (1997)

30. Amgoud, L., Cayrol, C.: A reasoning model based on the production of acceptable arguments. Ann. Math. Artif. Intell. 34(1-3), 197-215 (2002)

31. Bench-Capon, T.J.: Persuasion in practical argument using value-based argumentation frameworks. J. Logic Comput. 13(3), 429-448 (2003)

32. Kaci, S., Labreuche, C.: Argumentation framework with fuzzy preference relations. In: 13th International Conference on Information Processing and Management of Uncertainty, pp. 554-563 (2010) 
33. Martinez, D.C., Garcia, A.J., Simari, G.R.: An abstract argumentation framework with varied-strength attacks. In: International Conference on Principles of Knowledge Representation and Reasoning, pp. 135-143 (2008)

34. Janssen, J., De Cock, M., Vermeir, D.: Fuzzy argumentation frameworks. In: Information Processing and Management of Uncertainty in Knowledge-based Systems, pp. 513-520, June 2008

35. Zadeh, L.A.: Fuzzy sets. Inf. Control 8(3), 338-353 (1965)

36. Zadeh, L.A.: Fuzzy Sets, Fuzzy Logic, Fuzzy Systems. World Scientific Press (1966)

37. Li, H., Oren, N., Norman, T.J.: Probabilistic argumentation frameworks. In: Modgil, S., Oren, N., Toni, F. (eds.) TAFA 2011. LNCS (LNAI), vol. 7132, pp. 1-16. Springer, Heidelberg (2012). doi:10.1007/978-3-642-29184-5_1

38. Matt, P.-A., Toni, F.: A game-theoretic measure of argument strength for abstract argumentation. In: Hölldobler, S., Lutz, C., Wansing, H. (eds.) JELIA 2008. LNCS (LNAI), vol. 5293, pp. 285-297. Springer, Heidelberg (2008). doi:10.1007/ 978-3-540-87803-2_24

39. Vreeswijk, G.: Defeasible dialectics: a controversy-oriented approach towards defeasible argumentation. J. Logic Comput. 3, 3-27 (1993)

40. Baroni, P., Caminada, M., Giacomin, M.: An introduction to argumentation semantics. Knowl. Eng. Rev. 26(4), 365-410 (2011)

41. Wu, Y., Caminada, M., Podlaszewski, M.: A labelling based justification status of arguments. Stud. Logic 3(4), 12-29 (2010). 13th International Workshop on Non-Monotonic Reasoning

42. Dung, P.M., Mancarellab, P., Toni, F.: Computing ideal sceptical argumentation. Artif. Intell. 171(10-15), 642-674 (2007)

43. Caminada, M.W.A.: A labelling approach for ideal and stage semantics. Argument Comput. 2(1), 1-21 (2006)

44. Caminada, M.W.A., Carnielli, W.A., Dunne, P.E.: Semi-stable semantics. J. Logic Comput. 22(5), 1207-1254 (2012)

45. Caminada, M.W.A.: An algorithm for stage semantics. In: Baroni, P., Cerutti, F., Giacomin, M., Simari, G.R. (eds.): Frontiers in Artificial Intelligence and Applications, Proceedings of the 3rd International Conference on Computational Models of Argument (COMMA 2010), vol. 216, pp. 147-158. IOS Press (2010)

46. Jakobovits, H., Vermeir, D.: Robust semantics for argumentation frameworks. Logic. Comput. 9(2), 215-261 (1999)

47. Baroni, M., Giacomin, M., Guida, G.: Scc-recursiveness: a general schema for argumentation semantics. Artif. Intell. 168(1-2), 165-2010 (2005)

48. Baroni, P., Giacomin, M.: Semantics of abstract argument systems. In: Simari, G., Rahwan, I. (eds.): Argumentation in Artificial Intelligence, pp. 25-44. Springer, Heidelberg (2009)

49. Konieczny, S., Marquis, P., Vesic, S.: On supported inference and extension selection in abstract argumentation frameworks. In: Destercke, S., Denoeux, T. (eds.) ECSQARU 2015. LNCS (LNAI), vol. 9161, pp. 49-59. Springer, Heidelberg (2015). doi:10.1007/978-3-319-20807-7_5

50. Coste-Marquis, S., Konieczny, S., Marquis, P., Akli Ouali, M.: Selecting extensions in weighted argumentation frameworks. In: Computational Models of Argument, COMMA (2012)

51. Bryant, D., Krause, P.: A review of current defeasible reasoning implementations. Knowl. Eng. Rev. 23(3), 227-260 (2008)

52. Fox, J., Glasspool, D., Grecu, D., Modgil, S., South, M., Patkar, V.: Argumentation-based inference and decision making-a medical perspective. IEEE Intell. Syst., 21-23 (2007) 
53. Fox, J., Black, L., Glasspool, D., Modgil, S., Oettinger, A., Patkar, V., Williams, M.: Towards a general model for argumentation services. In: AAAI Spring Symposium Series (2006)

54. Glasspool, D., Fox, J., Oettinger, A., Smith-Spark, J.: Argumentation in decision support for medical care planning for patients and clinicians. In: AAAI Spring Symposium: Argumentation for Consumers of Healthcare, pp. 58-63 (2006)

55. Pollock, J.L.: Defeasible reasoning with variable degrees of justification. Artif. Intell. 133, 233-282 (2001)

56. Chang, C.F., Miller, A., Ghose, A.: Mixed-initiative argumentation: group decision support in medicine. In: Kostkova, P. (ed.) eHealth 2009. LNICSSITE, vol. 27, pp. 43-50. Springer, Heidelberg (2010). doi:10.1007/978-3-642-11745-9_8

57. Grando, M.A., Moss, L., Sleeman, D., Kinsella, J.: Argumentation-logic for creating and explaining medical hypotheses. Artif. Intell. Med 58(1), 1-13 (2013)

58. Gorogiannis, N., Hunter, A., Patkar, V., Williams, M.: Argumentation about treatment efficacy. In: Riaño, D., Teije, A., Miksch, S., Peleg, M. (eds.) KR4HC 2009. LNCS (LNAI), vol. 5943, pp. 169-179. Springer, Heidelberg (2010). doi:10.1007/ 978-3-642-11808-1_14

59. Williams, M., Hunter, A.: Harnessing ontologies for argument-based decisionmaking in breast cancer. In: ICTAI (2), pp. 254-261 (2007)

60. Longo, L., Hederman, L.: Argumentation theory for decision support in healthcare: a comparison with machine learning. In: Imamura, K., Usui, S., Shirao, T., Kasamatsu, T., Schwabe, L., Zhong, N. (eds.) BHI 2013. LNCS (LNAI), vol. 8211, pp. 168-180. Springer, Heidelberg (2013). doi:10.1007/978-3-319-02753-1_17

61. Tolchinsky, P., Cortes, U., Modgil, S., Caballero, F., Lopez-Navidad, A.: Increasing human-organ transplant availability: argumentation-based agent deliberation. IEEE Intell. Syst. 21(6), 30-37 (2006)

62. Patkar, V., Hurt, C., Steele, R., Love, S., Purushotham, A., Williams, M., Thomson, R., Fox, J.: Evidence-based guidelines and decision support services: a discussion and evaluation in triple assessment of suspected breast cancer. Br. J. Cancer 95(11), 1490-1496 (2006)

63. Fox, J., Das, S.: Safe and Sound: Artificial Intelligence in Hazardous Applications, 1st edn. AAAI Press (2000)

64. Hunter, A., Williams, M.: Argumentation for aggregating clinical evidence. In: ICTAI (1), pp. 361-368 (2010)

65. Prakken, H.: Ai \& law, logic and argument schemes. Argumentation (Special Issue on The Toulmin Model Today) 19, 303-320 (2005)

66. Jones, G.R.: Organizational Theory, Design, and Change: Text and Cases, 6th edn. Pearson Prentice Hall, Upper Saddle River, NJ (2010)

67. Daft, R.L.: Organization Theory and Design, 9th edn. Thomson South-Western, Mason, OH (2007)

68. Rapoport, A.: Decision Theory and Decision Behaviour. Springer (1989)

69. Longo, L., Rusconi, F., Noce, L., Barrett, S.: The importance of human mental workload in web-design. In: 8th International Conference on Web Information Systems and Technologies, pp. 403-409, April 2012

70. Longo, L.: Formalising human mental workload as non-monotonic concept for adaptive and personalised web-design. In: Masthoff, J., Mobasher, B., Desmarais, M.C., Nkambou, R. (eds.) UMAP 2012. LNCS, vol. 7379, pp. 369-373. Springer, Heidelberg (2012). doi:10.1007/978-3-642-31454-4_38 
71. Longo, L., Dondio, P.: On the relationship between perception of usability and subjective mental workload of web interfaces. In: IEEE/WIC/ACM International Conference on Web Intelligence and Intelligent Agent Technology, WI-IAT 2015, Singapore, December 6-9, vol. I, pp. 345-352 (2015)

72. Dondio, P., Longo, L.: Trust-based techniques for collective intelligence in social search systems. In: Bessis, N., Xhafa, F. (eds.) Next Generation Data Technologies for CCI, SCI, vol. 352, pp. 113-135. Springer, Heidelberg (2011)

73. Longo, L., Dondio, P., Barrett, S.: Enhancing social search: a computational collective intelligence model of behavioural traits, trust and time. Trans. Comput. Collective Intell. 2, 46-69 (2010)

74. Luca, L., Stephen, B., Pierpaolo, D.: Information foraging theory as a form of collective intelligence for social search. In: Nguyen, N.T., Kowalczyk, R., Chen, S.M. (eds.) ICCCI 2009. LNCS (LNAI), vol. 5796, pp. 63-74. Springer, Heidelberg (2009). doi:10.1007/978-3-642-04441-0_5

75. Možina, M., Žabkar, J., Bratko, I.: Argument based machine learning. Artif. Intell. 171(10-15), 922-937 (2007). Argumentation in Artificial Intelligence

76. Lippi, M., Torroni, P.: Argument mining: a machine learning perspective. In: Black, E., Modgil, S., Oren, N. (eds.) TAFA 2015. LNCS (LNAI), vol. 9524, pp. 163-176. Springer, Heidelberg (2015). doi:10.1007/978-3-319-28460-6_10 